\title{
Tabletop X-rays light up
}

\section{Compact device promises to open window on chemical reactions in the lab.}

\section{BY KATHERINE BOURZAC}

$\mathrm{T}$ he pressurized, cylindrical chamber fits in the palm of Margaret Murnane's hand. Yet out of one end of the device comes an X-ray beam that packs almost as much punch as the light generated by massive particle accelerators.

Murnane and Henry Kapteyn, both physicists at JILA in Boulder, Colorado, a joint institute of the University of Colorado and the US National Institute of Standards and Technology, have reported the first tabletop source of ultra-short, laser-like pulses of low energy, or 'soft', X-rays. The light, capable of probing the structure and dynamics of molecules, was previously available only at large, billion-dollar national facilities such as synchrotrons or free-electron lasers, where competition for use of the equipment is fierce. But the report by Murnane, Kapteyn and their colleagues, published in the 8 June issue of Science (T. Popmintchev et al. Science 336, 1287-1291; 2012), suggests that the devices might soon lie within the grasp of a university laboratory budget. "For us, it's incredible that we can do this at all in a tabletop system," says Murnane. "Three years ago, people would have said 'only large facilities can do that'."

Murnane and Kapteyn, a husband-and-wife team who also head the Boulder-based company KMLabs, already sell a similar tabletop source of extreme-ultraviolet light. Murnane thinks that a future soft X-ray source should cost about US\$1 million, and hopes that its relatively low cost and small size will open up X-ray studies for materials scientists, biologists and others. The beams generated by the device could, for instance, help materials scientists to make better solar materials by tracking the paths of electrons through solar cells, and might allow chemists to trace the ultrafast dynamics of photosynthesis and catalysis. "This is something people have been waiting for for a very long time," says Oleg Shpyrko, a physicist at the University of California, San Diego. Shpyrko often waits months to get his experiments accepted at the Advanced Photon Source, a synchrotron at Argonne National Laboratory in Illinois, and must then fly his students halfway across the country to do the work.

The tabletop sources rely on a technique called high-harmonic generation, in which laser light is passed through a medium that converts it to light of shorter wavelengths and higher frequencies. Shine a ruby laser into a quartz crystal, for example, and a beam of ultraviolet light comes out - albeit dimmer, but still focused like a laser beam.

with a wavelength of 0.8 nanometres. Around these wavelengths, many of the chemical elements used in magnets and superconductors absorb a characteristic band of light. Kapteyn says that this could be used by chemists to discern the spin states of nickel atoms that make up the bits of information in magnetic computer hard drives, for example.

The tabletop systems have already surpassed the larger light-source facilities in terms of laser pulse speed. Murnane and Kapteyn's device produces very fast pulses, as short as 2.5 attoseconds $\left(10^{-18}\right.$ seconds $)$ - faster than the picosecond $\left(10^{-12}\right)$ pulses of synchrotrons and the femtosecond $\left(10^{-15}\right)$ pulses of freeelectron lasers. That timescale is even quicker than the making and breaking of chemical bonds. "At this timescale, we can start using these light sources to address questions where we have no idea what the answers will be," says Ferenc Krausz, a physicist at the Max Planck Institute for Quantum Optics in Garching, Germany, and co-founder of Femtolasers, a company that sells ultrafast light sources.

Light produced by these sources is much less intense than that at the big national facilities, however. That's one reason why physicist Emma Springate, who runs Artemis, part of the Central Laser Facility at the Science and Technology Facilities Council's Rutherford Appleton Laboratory near Didcot, UK, wants access to

Murnane and Kapteyn have pushed highharmonic generation to its limits, with a system that uses an infrared laser as the source and pressurized helium gas as the medium. The laser creates a strong electric field, which draws electrons away from the helium atoms, allowing the electrons to absorb energy from the electric field. When they slam back in to the helium atoms, they release that absorbed energy as shorter-wavelength photons - but only about one photon comes out for every 5,000 infrared photons that are put in.

The result is light at wavelengths almost as short as those delivered by synchrotrons. By increasing the pressure in the gas - something theorists thought might defocus the light beam - Murnane and Kapteyn can produce light 